\title{
Simulation and verification for seed-filling performance of cell wheel precision seed metering device based on discrete element method
}

\author{
Qinghui Lai, Ziwu $\mathrm{Hu}^{\mathrm{a}}$, Jinlong Xing and Wenpeng Ma \\ Faculty of Modern Agriculture Engineering, Kunming University of Science \& Technology, Kunming, China
}

\begin{abstract}
The cell wheel seed metering device was improved and a stirring seed-filling device was added to improve the seed-filling performance of cell wheel pseudo-ginseng precision seed metering devices. Using pseudo-ginseng seeds in Wenshan Prefecture, Yunnan Province as the objects for seed metering, the software application EDEM was adopted based on the discrete element method for the simulation calculation and analysis of the seed-filling performance of the seed metering device under 4 rotational speeds of the cell wheel and 6 rotational speeds of the stir wheel. The simulation results indicate that the filling ratio increases as the rotational speed of the stir wheel increases under a constant rotational speed of the cell wheel. Test verification of the simulation analysis results was conducted on the test bed of the seed metering device. The results indicate that increasing the rotational speed of the stir wheel can obtain a filling ratio of over $90 \%$. The test results display a similar variation trend to that of the simulation analysis with an error of average filling ratio less than $5 \%$. Therefore, it is feasible to analyze the seed-filling performance of the stirring and seed-filling device of the seed metering device with the discrete element method.
\end{abstract}

\section{Introduction}

Pseudo-ginseng is a rare traditional Chinese medicinal herb. Its seeds have irregular shapes with high internal friction among themselves, and mis-seeding happens frequently during the seeding of pseudoginseng, therefore improving seeding yield has always been a research focus of the pseudo-ginseng precision seed metering device. The cell wheel seed metering device has been widely adopted for the seeding of various crops ${ }^{[1-5]}$. However, the cell wheel seed metering device has strict requirements for the shape and size of seeds. Its filling ratio is significantly affected by operating speed and its operating efficiency is very low. Many researchers have conducted studies on the cell wheel seed metering device to improve its seed-filling performance. P. P. Garcia ${ }^{[6]}$ found by studies that the seed metering precision is under significant influence of the hole shape and size of the cell wheel seed metering device. $\mathrm{W} . \mathrm{Hu}^{[7]}$ optimized the hole parameters of the seed metering device according to the size of seeds. L. X. Wen et al. ${ }^{[8]}$ designed an elastic follow-up seed protection device for the hole wheel seed metering device to reduce the damage of rice seeds and the wear on the outside surface of the cell wheel. Y. J. Qun et al. ${ }^{[9]}$ conducted a discrete element simulation analysis on the performance of the hole wheel soybean seed metering device with their self-developed 3D software application

\footnotetext{
${ }^{a}$ Corresponding author : m15288451629@163.com
}

(C) The Authors, published by EDP Sciences. This is an open access article distributed under the terms of the Creative Commons Attribution License 4.0 (http://creativecommons.org/licenses/by/4.0/). 
CAE and verified its feasibility. T. C. Zhou et al. ${ }^{[10]}$ designed a variable volume hole wheel seed metering device composed of the hole wheel and adjustment ring (tongue) and conducted tests on its performance. By collecting the sample video files of the corn seed flow at the orifice of the cell wheel seed metering device, Q. Ling ${ }^{[11]}$ adopted image processing for the statistics of the performance indexes of the seed metering device. W. Z. Man et al. ${ }^{[12]}$ acquired the seed-filling process of the hole rice seed metering wheel by high-speed photography and analyzed the flow regularity, status and seed-filling trend of rice seeds in the seed-filling area. L. Tao et al. ${ }^{[13]}$ studied the influence of 3 types of hole structures on the seed group disturbance and seed metering performance of the cell wheel rape seed metering device.

The analysis on the performance of the seed metering device by conventional tests costs a long period of time and lots of manpower and material resources. With the development of computer technology, the computer-aided analysis software application EDEM has been widely adopted in the simulation analysis of particulate matters. S. Maitraye et al. ${ }^{[14]}$ conducted a numerical simulation analysis on the prilling technique of fluidized bed by coupling CFD-DEM-PBM. L. Bart et al. ${ }^{[15]}$ conducted a simulation analysis on the separation process of the straw combine harvester by the particle model of flexible straw discrete elements. Z. Xuan et al. ${ }^{[16]}$ studied the flow process of soybean in the chute by the discrete element method. I. Keppler et al. ${ }^{[17]}$ conducted a simulation analysis on the flow of wheat in the mixed gas grain dryer by the discrete element method. L. Y. Qin et al. $^{[18]}$ simulated the air-suction seed metering device of the soybean no-tillage planter by the discrete element method and optimized the working parameters by the response surface methodology.

Starting with the seed-filling performance of the seed metering device, this paper improved the structure of the cell wheel seed metering device to deal with its low seed metering efficiency of pseudo-ginseng seeds. A contact model among the pseudo-ginseng seed particles in the seed case was established by the discrete element theory to analyze the normal force and tangential force from the impact contact among the particles so as to determine the principal factor that affects the seed group disturbance. The influence of the rotational speed of the stir wheel and that of the cell wheel on the filling ratio was investigated with the discrete element numerical simulation software application EDEM to analyze the fluctuation of the average normal force and the average speed of the seed group with time, as well as the variation trend of the filling area with the rotational speed of the stir wheel. The simulation analysis results were verified by tests to determine the feasibility of the discrete element simulation analysis method. The simulation analysis on the seed-filling performance of the cell wheel seed metering device by discrete element numerical simulation can save test costs, reduce the test cycle and provide references for the improvement of pseudo-ginseng precision planters.

\section{Discrete element theory}

\subsection{Establishment of contact model}

The discrete element method simulates the propagation of movement in a particulate set. When the seed metering device is in operation, the seeds in the seed case are disturbed by the rotation of the cell wheel, and the movement of seeds will certainly cause impact among seeds, thus generating contact force $^{[19,20]}$. In the discrete element analysis method, the contact model can be divided into soft particle contact and hard particle contact according to different contact forms. The soft particle contact model allows a certain period of impact, and it calculates the contact force according to the impact overlap and tangential displacement of particles by taking into account the impact of multiple particles. Such contact force is closely correlated with seed group disturbance. Considering there is no surface adhesion on pseudo-ginseng seeds, the Hertz-Mindlin soft particle contact model was adopted ${ }^{[21-23]}$. The contact of this model was simplified as the damped oscillation of the spring oscillator with couplers and slides ${ }^{[17,24,25]}$ as shown Figure 1. Its differential equation is: 


$$
m \cdot \frac{d^{2} x}{d t^{2}}+c \cdot \frac{d x}{d t}+k x=0
$$

Where: $m$ stands for the mass of model particles; $x$ stands for the displacement deviated from the equilibrium position; $c$ stands for the contact damping coefficient; and $k$ stands for the elastic coefficient of the spring.

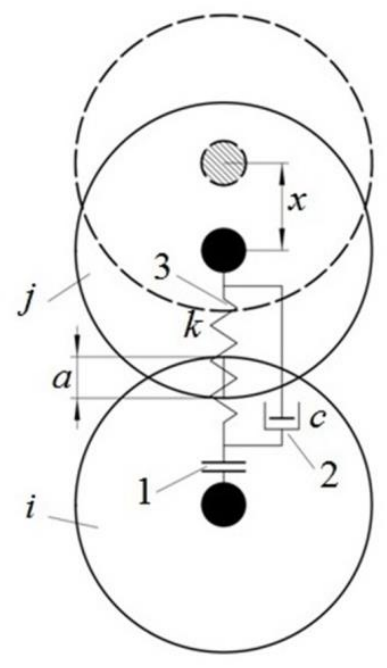

a. Normal contact model

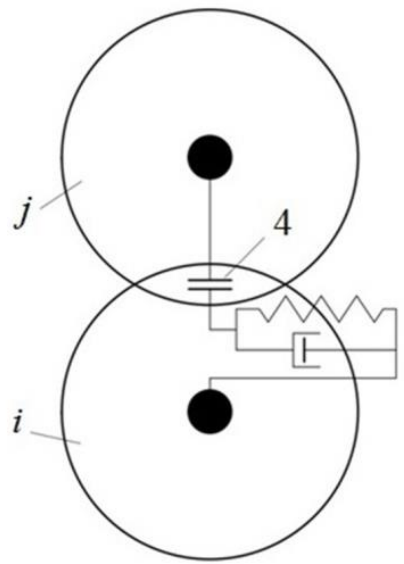

b. Tangential contact model

Figure 1. Simplified soft spherical model of contact force among particles.

1. Coupler 2. Damper 3. Spring 4. Slider

Note: $i$ stands for particle $i ; j$ stands for particle $j$

On the normal direction, the coupler determines the pairing of contacted particles and no force is introduced; on the tangential direction, the slider can realizes the slide of two particles by the normal force and friction force ${ }^{[26]}$.

\subsection{Analysis of contact force}

In the soft spherical particle impact model, the contact force can be divided into the normal force and tangential force, and the normal force is the combination of the elastic force and damping force in the model. As for the 3D spherical particles of pseudo-ginseng seeds, according to the Hertz elastic contact theory, the normal force $F_{n}$ and the tangential force $F_{t}$ can be expressed as follows:

$$
\begin{gathered}
F_{n}=\left(-k_{n} a^{3 / 2}-c_{n} v_{i j} \cdot n\right) n \\
F_{t}=-k_{t} \delta-c_{t} v_{c t}
\end{gathered}
$$

Where: $k_{n}$ stands for the modulus of normal elasticity; $k_{t}$ stands for the modulus of tangential elasticity; $c_{n}$ stands for the normal damping coefficient; $c_{t}$ stands for the tangential damping coefficient; $a$ stands for the normal overlap; $v_{i j}$ stands for the relative speed of particle $i$ to particle $j$; $v_{c t}$ stands for the sliding speed of the contact point; $\delta$ stands for the tangential displacement; $n$ stands for the normal unit vector of the contact surface between particle $i$ and particle $j$.

If the following formula is tenable:

$$
\left|F_{t}\right| \geq \mu\left|F_{n}\right|
$$


Relative sliding occurs between particle $i$ and particle $j$, then:

$$
F_{t}=-\mu_{s}\left|F_{n}\right| \cdot n_{t}
$$

Where: $\mu_{s}$ stands for the static friction factor which is determined by the physical properties of pseudo-ginseng seeds; $n_{t}$ stands for the tangential unit vector. According to formula (5), the internal friction force among seeds is the tangential force in the above contact model. The tangential force is determined by the normal force and is also a principal factor of seed group disturbance.

The internal friction force among pseudo-ginseng seeds is the principal factor that hinders the relative movement or the trend of relative movement among seeds. The internal friction force among pseudo-ginseng seeds is greater and the relative movement is weaker, which are not conducive to the seed-filling of the seed metering device. Therefore, the stirring and seed-filling device added to the cell wheel seed metering device can overcome the friction force among seeds, facilitate the relative movement among seeds and improve the seed-filling efficiency.

\section{Discrete element simulation analysis on seed-filling process}

\subsection{Structure and working principle of seed metering devices}

The structure of the seed metering device is as shown in Figure 2. It mainly consists of a seed cleaner, a cell wheel, a seed case, a stir wheel, a hairbrush, a seed protecting plate, a transmission shaft and a shell. There are cell wheel holes with the diameter of $8.0 \mathrm{~mm}$ and the depth of $5.5 \mathrm{~mm}^{[27]}$. The simplestructured seed metering device (176 mm long, $42 \mathrm{~mm}$ wide and $240 \mathrm{~mm}$ high) is light in mass and applicable to the seeding of pseudo-ginseng which requires narrow row space. During operation, the cell wheel of the seed metering device rotates clockwise while the stir wheel rotates anticlockwise. The seed group in the seed case moves with the stir wheel, and the seeds rotate with the cell wheel when they fall into the holes of the cell wheel in the seed-filling area. The excessive seeds will be removed by the hairbrush when reaching it. Then the seeds pass the seed protecting area to the seed charging area. At that time, the seeds detach from the holes by their own gravity, and those cannot fall themselves will be forced out of the holes by the seed cleaner.

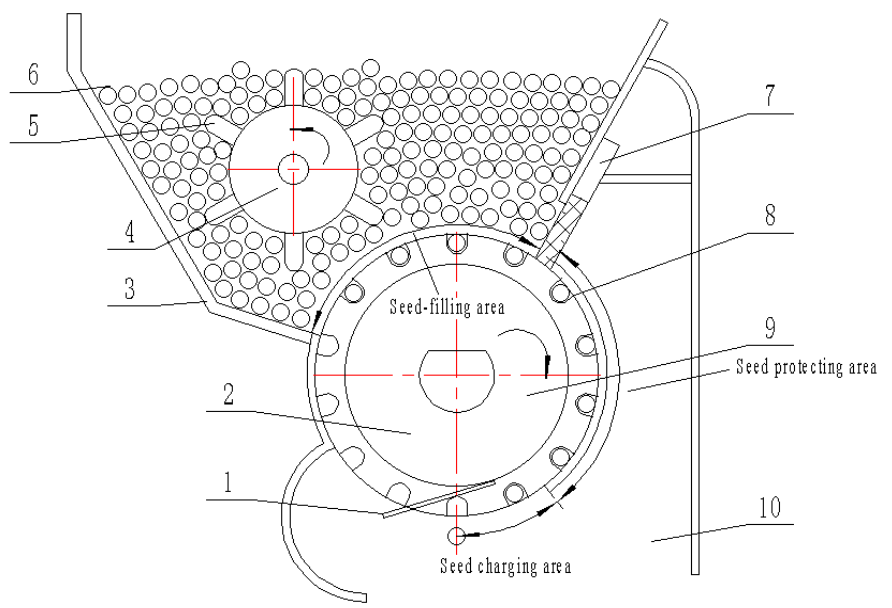

Figure 2. Structure and principle of the seed metering device.

1. Seed cleaner 2. Cell wheel 3. Seed case 4. Stir wheel shaft 5. Stir wheel 6.seeds

7. Hairbrush 8 . Seed protecting plate 9. Seed metering shaft 10. Shell 
Considering the triaxial size and external physical characteristics of pseudo-ginseng seeds, spheres with different diameters were used for filling and superposition by the discrete element analysis software application EDEM to make them more close to the geometrical size of real seeds ${ }^{[28]}$. The pseudo-ginseng seed model was established by filling and superposition of 5 spheres in the discrete element software application EDEM as shown in Figure 3.

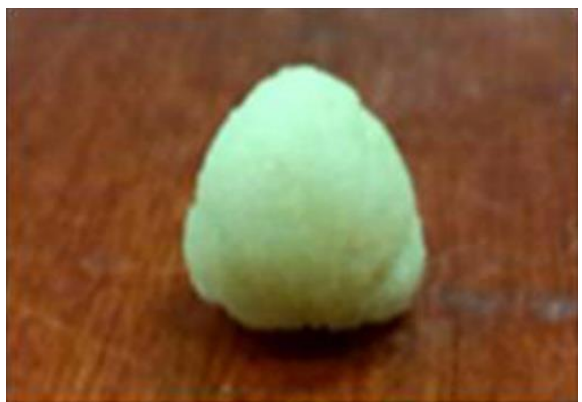

a. Real pseudo-ginseng seed

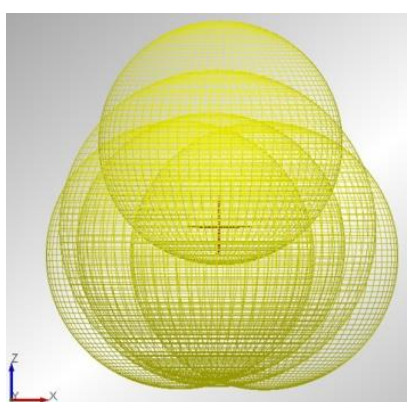

b. discrete element model of pseudo-ginseng seeds

Figure 3. Model of pseudo-ginseng seeds.

The Hertz-Mindlin (no slip) contact model was adopted between seed particles as well as between seeds and the seed metering device, and the particle properties and contact parameters were set up according to Table $1^{[29]}$. The generation type of particles was set as dynamic, the mass of the generated particle was $150 \mathrm{~g}$ and the particle size complies with the normal distribution. The step length of simulation time, simulation time and mesh size were set as $1 \mathrm{e}-5 \mathrm{~s}, 20 \mathrm{~s}$ and $2 \mathrm{~mm}$ respectively.

Table 1. Parameters needed for simulation.

\begin{tabular}{|c|c|}
\hline Parameter & Value \\
\hline Particle density $/ \mathrm{kg} \cdot \mathrm{m}^{-3}$ & 1100 \\
\hline Poisson's ratio of particles & 0,4 \\
\hline Shear modulus of particles/MPa & $1,3 \times 10^{7}$ \\
\hline Recovery coefficient among particles & 0,48 \\
\hline Static friction coefficient among particles & 0,32 \\
\hline Rolling friction coefficient among particles & 0,085 \\
\hline
\end{tabular}

With the filling ratio as the test index, 6 rotational speeds of the stir wheel $(0,50,100,150,200$ and $250 \mathrm{rpm})$ were selected to conduct 3 repeated simulation tests on 4 rotational speeds of the cell wheel $(60,80,100$ and 120rpm). Each group of the test tested 200 holes continuously, and it shall be deemed as miss-filling when there are no seeds in holes in the seed protecting area. After obtaining the statistics of the number of miss-filled holes, the filling ratio A and the average filling ratio is were calculated according to formula 6 .

$$
A=\left(1-\frac{N_{m}}{N}\right) \times 100 \%
$$

Where: Nm stands for the number of miss-filled holes, and $\mathrm{N}$ stands for the number of continuously tested holes, $\mathrm{N}=200$.

\subsection{Simulation test results and analysis}

\subsubsection{Influence of rotational speed of stir wheel on filling ratio}


Figure 4 shows the variation trend of the filling ratio with the rotational speed of the stir wheel under 4 rotational speeds of the cell wheel. It can be seen from the figure that the filling ratio under each rotational speed of the cell wheel is on the rise as the rotational speed of the stir wheel increases. The maximum filling ratio is $99,50 \%$ when the rotational speed of the cell wheel reaches $60 \mathrm{rpm}$, and the filling ratio can still reach $91,33 \%$ when the rotational speed of the cell wheel grows to $120 \mathrm{rpm}$. The filling ratio decreases as the rotational speed of the cell wheel increases when the rotational speed of the stir wheel is constant.

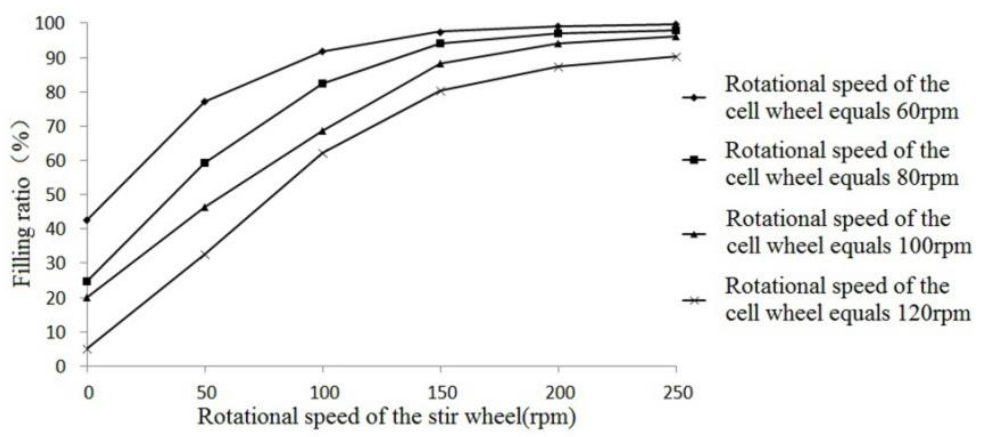

Figure 4. Variation trend of the filling ratio with the rotational speed of the stir wheel under different rotational speeds of the cell wheel.

\subsubsection{Influence of rotational speed of stir wheel on seed group disturbance}

Figure 5 and Figure 6 shows the fluctuation of the average normal force of contact among pseudoginseng seed particles and the average speed of particles within the range of $10 \mathrm{~s}$ to $11 \mathrm{~s}$ under different rotational speeds of the cell wheel when the rotational speed of the cell wheel is 60rpm. It can be known from Figure 5 that the average normal force fluctuates irregularly with time under each rotational speed of the stir wheel, and it fluctuates more wildly as the rotational speed of the stir wheel increases. When the rotational speed of the stir wheel is 0 , the average normal force among pseudoginseng seed particles fluctuate the least with time with an average value of $0,0028 \mathrm{~N}$. When the rotational speed of the stir wheel exceeds $50 \mathrm{rpm}$, the average normal force increases significantly with little difference in the average value (around $0,02 \mathrm{~N}$ ) when the rotational speed of the stir wheel lies between 50rpm and 250rpm. According to Figure 6, the average speed of pseudo-ginseng seed particles increases as the rotational speed of the stir wheel grows. It can be known from Figure 4 to Figure 6 that a higher rotational speed of the stir wheel causes higher average speed of seeds, seed group disturbance and filling ratio, indicating that increasing the rotational speed of the stir wheel can help seeds get into the holes to complete the seed-filling process.

\subsubsection{Influence of rotational speed of stir wheel on filling area}

The filling area is an area formed by the overlap of movement tracks of seeds in the seed-filling area of the seed metering device. A larger filling area indicates lower filling efficiency and higher filling efficiency otherwise. Figure 7 shows the filling area under different rotational speeds of the stir wheel by tracking the movement tracks of seed groups when the rotational speed of the cell wheel is 60rpm. According to Figure 7, the filling area decreases as the rotational speed of the stir wheel increases. When the rotational speed of the stir wheel is 0 , the filling area occupies the entire seed-filling area of the seed metering device and seeds are able to enter the holes anywhere in the seed-filling area, thus the seed-filling efficiency is the lowest at that time. When the rotational speed of the stir wheel is $250 \mathrm{rpm}$, the filling area is mainly concentrated at the initial position of the seed-filling area and the holes can finish seed-filling at the initial position, thus the seed-filling efficiency is the highest. 


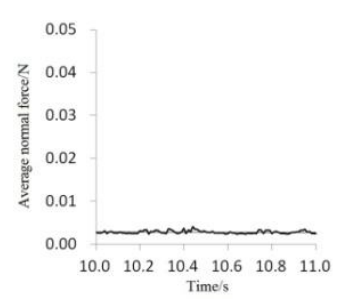

a. Rotational speed of the stir wheel equals 0

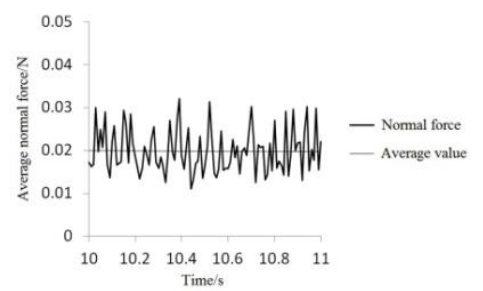

d. Rotational speed of the stir wheel equals $150 \mathrm{rpm}$

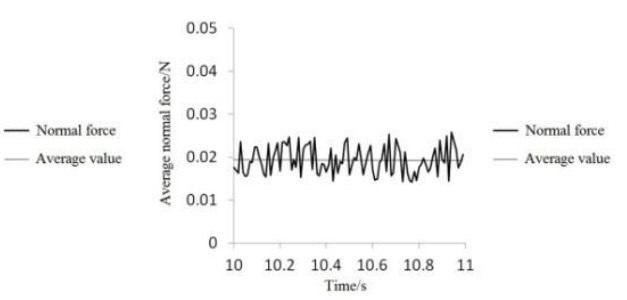

b. Rotational speed of the stir wheel equals $50 \mathrm{rpm}$

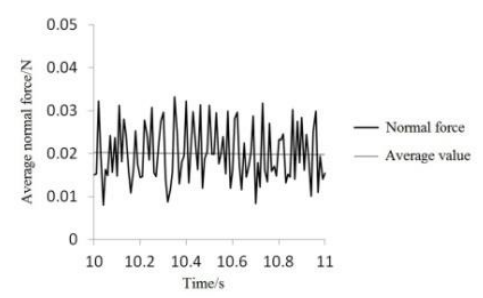

e. Rotational speed of the stir wheel equals $200 \mathrm{rpm}$

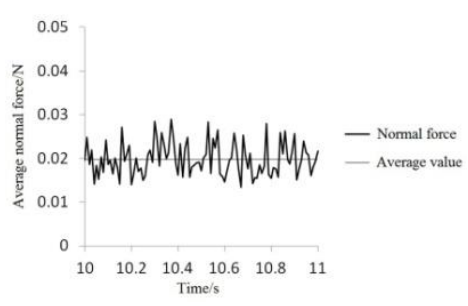

c. Rotational speed of the stir wheel equals $100 \mathrm{rpm}$

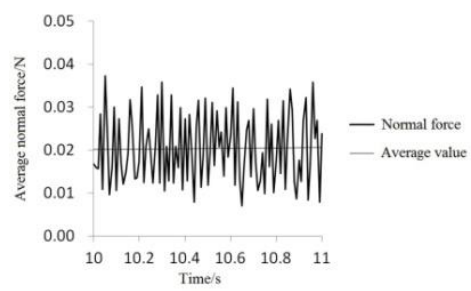

f. Rotational speed of the stir wheel equals $250 \mathrm{rpm}$

Figure 5. Fluctuation of the average normal force with time under different rotational speeds of the stir wheel.

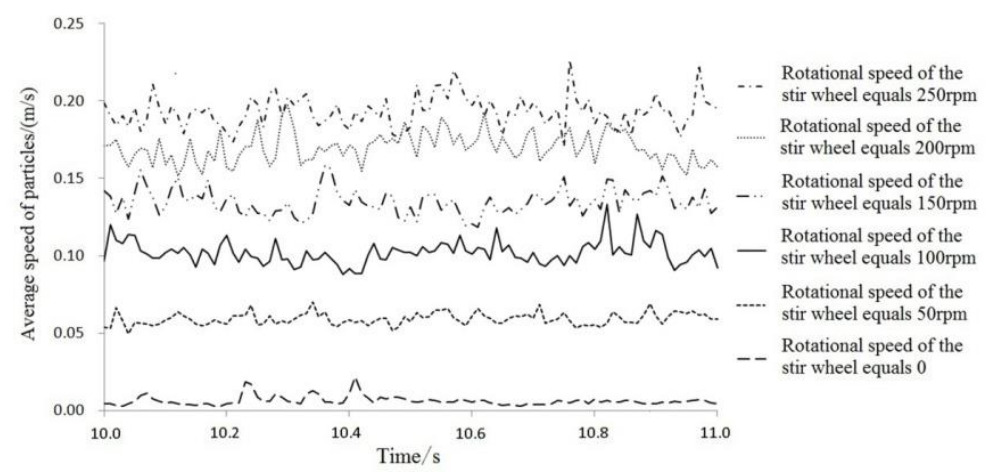

Figure 6. Fluctuation of the average speed of seeds with time under different rotational speeds of the stir wheel.

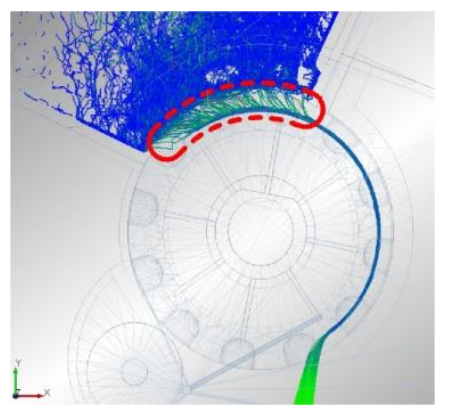

a. Rotational speed of the stir wheel equals 0

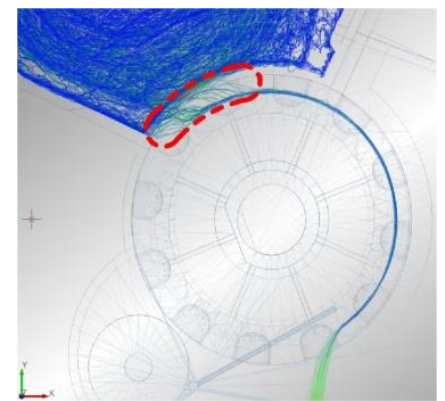

b. Rotational speed of the stir wheel equals 50rpm

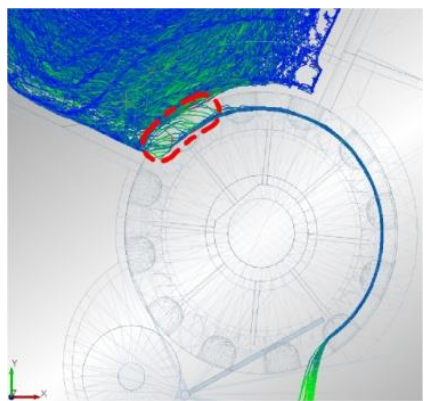

c. Rotational speed of the stir wheel equals $100 \mathrm{rpm}$ 


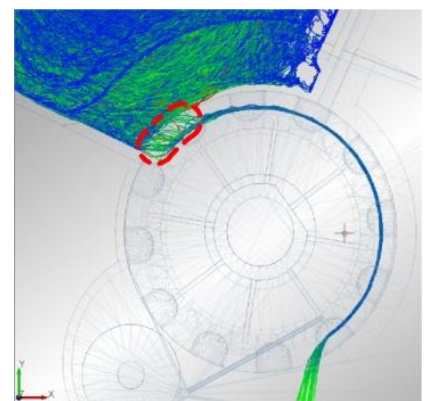

d. Rotational speed of the stir wheel equals $150 \mathrm{rpm}$

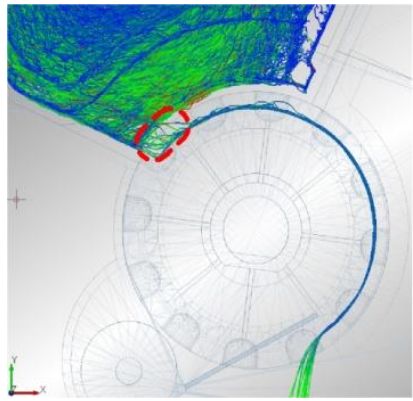

e. Rotational speed of the stir wheel equals $200 \mathrm{rpm}$

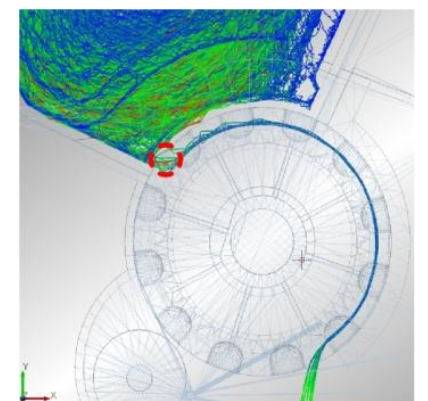

f. Rotational speed of the stir wheel equals $250 \mathrm{rpm}$

Figure 7. Variation of the filling area under different rotational speeds of the stir wheel.

\section{Test verification}

\subsection{Test materials and method}

\subsubsection{Test materials}

A test device of the seed metering device was produced according to the simulation model, and it was tested and verified on the test bed of the JPS-12 computer vision seed metering device. The pseudoginseng seeds picked from the pseudo-ginseng cultivation base in Wenshan Prefecture, Yunnan Province were selected as the test materials. The test facilities mainly include a DMK 23 G618 highspeed camera, a 57BYGH250C stepping motor, a TB6600 driver, a E30S4-1000-3-N-24 speed sensor, a ME204E electronic balance (with a weighing range of $0-220 \mathrm{~g}$ and the precision of $0.0001 \mathrm{~g}$ ) and a computer. As shown in Figure 8, the cell wheel is connected to the main shaft of the test bed through chain transmission and is driven by the electric motor to rotate. The output shaft of the stepping motor is connected to the left end of the stir wheel shaft and adjusts the rotational speed of the stir wheel by the driver. The speed sensor is connected to the right end of the stir wheel shaft to acquire the rotational speed of the stir wheel and to facilitate adjustment. The high-speed camera is mounted under the seed cleaning area of the seed metering device to record the images of seed metering. The rotational speeds of the cell wheel and the stir wheel as well as the recorded images can be displayed on the computer interface in real time.

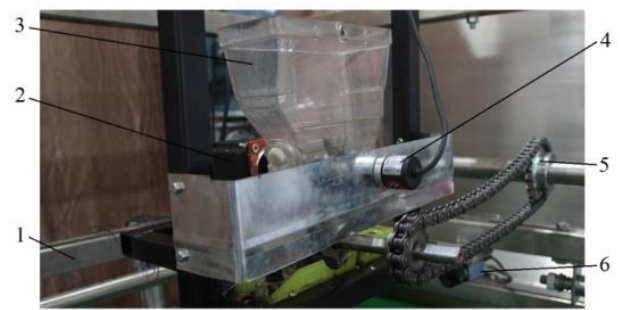

a. Test bed

1. Rack 2. Stepping motor 3. Seed metering device 4. Speed sensor 5. Chain transmission 6. High-speed camera

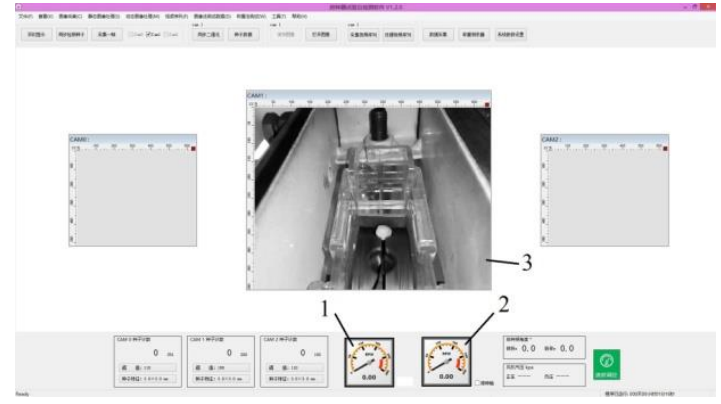

b. Display window of the computer

1. Display interface of the rotational speed of the stir wheel

2. Display interface of the rotational speed of the cell wheel

3. Display interface of the high-speed camera

Figure 8. Test setup. 


\subsubsection{Test method}

The 4 rotational speeds of the cell wheel and 6 rotational speeds of the stir wheel same as the simulation test were selected, and the filling ratio was adopted as the test index. Three repeated variance analyses were conducted on the test results by the two-factor equal repeated tests. Before each group of the test, weigh $150 \mathrm{~g}$ pseudo-ginseng seeds by the electronic balance and put them into the seed case and then proceed with the tests according to Table 2 . The images of the seed cleaning area were recorded with the high-speed camera. Circumstances that there are no seeds in the holes of the seed cleaning area should be deemed as miss-filling, and the filling ratio is calculated according to formula 6 . The filling ratio measured in each group of the test was recorded in Table 2.

Table 2. Design and results of the two-factor equal repeated tests.

\begin{tabular}{|c|c|c|c|c|c|}
\hline \multirow{2}{*}{ Test No. } & \multirow{2}{*}{$\begin{array}{c}\text { Rotational } \\
\text { speed of } \\
\text { the stir wheel } \\
\left(X_{1}\right) / \mathbf{r p m}\end{array}$} & \multirow{2}{*}{$\begin{array}{c}\text { Rotational } \\
\text { speed of } \\
\text { the cell wheel } \\
\left(X_{2}\right) / \mathbf{r p m}\end{array}$} & \multicolumn{3}{|c|}{ Filling Ratio $(A) / \%$} \\
\hline & & & 1 & 2 & 3 \\
\hline 1 & 0 & 60 & 43,5 & 47,5 & 42,5 \\
\hline 2 & 50 & 60 & 81,5 & 77,5 & 77,0 \\
\hline 3 & 100 & 60 & 92,0 & 92,0 & 84,0 \\
\hline 4 & 150 & 60 & 92,0 & 92,5 & 93,5 \\
\hline 5 & 200 & 60 & 96,5 & 94,5 & 94,5 \\
\hline 6 & 250 & 60 & 99,5 & 98,0 & 97,0 \\
\hline 7 & 0 & 80 & 28,0 & 26,0 & 24,5 \\
\hline 8 & 50 & 80 & 54,5 & 60,0 & 55,5 \\
\hline 9 & 100 & 80 & 81,0 & 81,0 & 79,0 \\
\hline 10 & 150 & 80 & 92,0 & 89,5 & 89,0 \\
\hline 11 & 200 & 80 & 95,0 & 91,5 & 93,5 \\
\hline 12 & 250 & 80 & 98,0 & 97,0 & 98,0 \\
\hline 13 & 0 & 100 & 15,0 & 18,0 & 20,0 \\
\hline 14 & 50 & 100 & 46,5 & 47,0 & 49,0 \\
\hline 15 & 100 & 100 & 71,5 & 71,5 & 72,0 \\
\hline 16 & 150 & 100 & 83,0 & 86,0 & 84,5 \\
\hline 17 & 200 & 100 & 89,5 & 90,0 & 92,5 \\
\hline 18 & 250 & 100 & 96,0 & 96,5 & 95,0 \\
\hline 19 & 0 & 120 & 7,0 & 9,0 & 6,5 \\
\hline 20 & 50 & 120 & 30,5 & 34,0 & 36,0 \\
\hline 21 & 100 & 120 & 58,5 & 55,5 & 56,0 \\
\hline 22 & 150 & 120 & 79,0 & 74,5 & 72,5 \\
\hline 23 & 200 & 120 & 89,5 & 87,0 & 81,5 \\
\hline 24 & 250 & 120 & 91,5 & 89,5 & 93,0 \\
\hline
\end{tabular}

\subsection{Test materials and method}

A variance analysis was conducted for the test results by the software application Design-Expert 8.0.6 to test its significance. According to Table 3, the $\mathrm{P}$ value of the rotational speed of the stir wheel $\left(X_{1}\right)$, $\mathrm{P}$ value of the rotational speed of the cell wheel $\left(X_{2}\right)$ and that of the interaction item $\left(X_{1} X_{2}\right)$ of the 
rotational speeds of the stir wheel and cell wheel are all less than 0.0001 , suggesting that the rotational speeds of the stir wheel and cell wheel as well as the interaction item of the rotational speeds of the stir wheel and cell wheel affect the filling ratio significantly.

Table 3. Variance analysis table of filling ratio $A$.

\begin{tabular}{|c|c|c|c|c|c|}
\hline Source & $\begin{array}{c}\text { Sum of } \\
\text { Squares }\end{array}$ & df & $\begin{array}{c}\text { Mean } \\
\text { Square }\end{array}$ & $\begin{array}{c}\text { F } \\
\text { Value }\end{array}$ & $\begin{array}{c}\text { P Value } \\
\text { Prob>F }\end{array}$ \\
\hline Model & 52956,38 & 23 & 2302,45 & 418,63 & $<0,0001^{* *}$ \\
\hline$X_{1}$ & 45008,08 & 5 & 9001,62 & 1636,66 & $<0,0001^{* *}$ \\
\hline$X_{2}$ & 5822,79 & 3 & 1940,93 & 352,90 & $<0,0001^{* *}$ \\
\hline$X_{1} X$ & 2125,50 & 15 & 141,70 & 25,76 & $<0,0001^{* *}$ \\
\hline Pure Error & 264,00 & 48 & 5,50 & & \\
\hline Cor Total & 53220,38 & 71 & & & \\
\hline
\end{tabular}

Note: “*” stands for significant $(\mathrm{P}<0,05)$ and “**” stands for extremely significant $(\mathrm{p}<0,01)$, similarly hereinafter.

Figure 9 shows the variation trend of the filling ratio with the rotational speed of the stir wheel under 4 rotational speeds of the cell wheel. It can be seen from the figure that the filling ratio grows as the rotational speed of the stir wheel increases, and it has a consistent variation trend with the test results of the simulation. The average error of the filling ratio of the simulation test and that of the verification test is $3,43 \%(<5 \%)$, indicating that it is feasible to analyze the seed-filling performance of the pseudo-ginseng precision seed metering device by the discrete element method ${ }^{[30]}$.

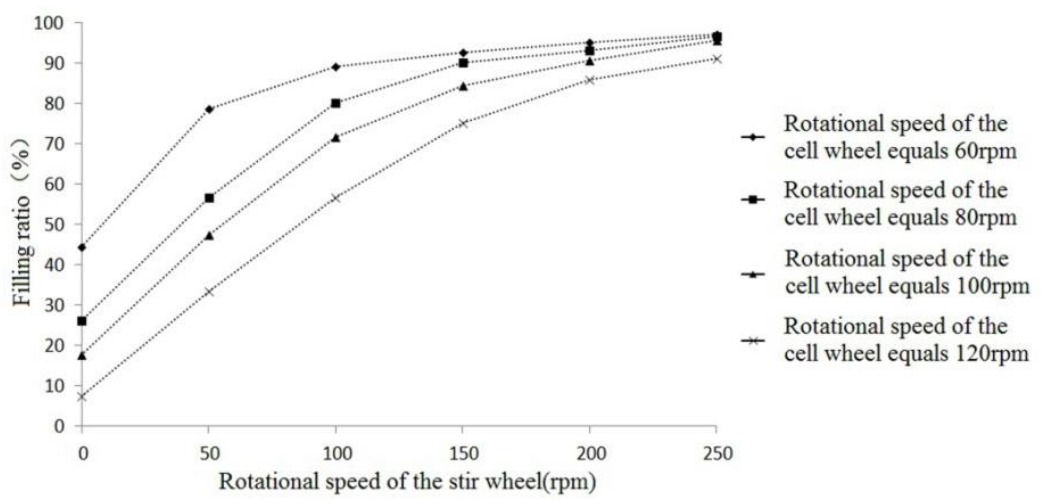

Figure 9. Variation trend of the filling ratio with the rotational speed of the stir wheel under different rotational speeds of the cell wheel.

\section{Conclusions}

(1) This paper conducted a theoretical analysis on the contact model of pseudo-ginseng seeds and proposed the stirring and seed metering method for the cell wheel seed metering device to overcome the friction force among seeds, improve the seed group disturbance, enhance the relative movement of seeds and improve the seed-filling efficiency. (2) The simulation test indicates that a higher rotational speed of the stir wheel causes higher seed group disturbance, average speed of seeds and filling ratio, thus easier for seeds to enter the holes. The filling area reduces as the rotational speed of the stir wheel increases. A larger filling area leads to lower filling efficiency while a smaller filling area leads to higher filling efficiency. When the filling area is minimal, it is indicated that the hole is filled in the 
initial position of the filling area, and the filling efficiency is the highest. When the filling area is maximum, it is indicated that the hole must pass through the whole filling area to have the seed fall into the hole, at this time, the filling efficiency is the lowest. (3) According to the variance analysis on the verification test results, the filling ratio is significantly affected by the rotational speeds of the cell wheel and the stir wheel as well as the interaction item of the rotational speeds of the cell wheel and the stir wheel. When the rotational speed of the cell wheel reaches the maximum, improving rotational speed of the stir wheel can cause a filling ratio of over $90 \%$. The test results are almost identical to the variation trend of the simulation analysis results with an average error of the filling ratio less than 5\% $(3,43 \%)$, indicating that it is feasible to analyze the seed-filling performance of the stirring and seedfilling device by the discrete element method. (4) The adding of a stirring and seed-filling device can improve the filling ratio of the cell wheel seed metering device so as to improve the seeding yield. When the seed metering filling ratio is guaranteed, adding a stirring and seed-filling device can improve the seeding metering efficiency of the seed metering device so as to increase the operating speed of planters.

\section{References}

1. Y.W. Sheng, W.C. You and J.C. Qian, Transactions of the CSAM, 40, 72 (2009)

2. Z.Y. Wen, Transactions of the CSAM, 36, 51 ( 2005)

3. W.M. Liang, G.C.Yun, G.X. Yan, et al., Transactions of the CSAE, 26, 119 (2010)

4. Y.W. Man, W.M. Lin and H.T. Feng. Journal of Agricultural Mechanization Research, 34 (2015)

5. W.Y. Yao, L.J. Dong, W.D. Wei, et al., Transactions of the CSAM, 43, 54 (2012)

6. P.P. Garcia, Participatory development of a corn seeder, Philippines: Philippines University (1993)

7. W. Hui, Philippines University (1991)

8. L.X. Wen, W.Z. Man, J.E. Chen, et al., Transactions of the CSAM, 39, 60 (2008)

9. Y.J. Qun, W. Gang, X. Nan and F. Hong. Transactions of the CSAM, 42, 83 (2011)

10. T.C. Zhou, L.H. Feng, W.M. Liang, et al., Transactions of the CSAM, 26, 114 (2010)

11. Q. Ling, Agricultural Technology \& Equipment, 23 (2014)

12. W.Z. Man, L.X. Wen, H.S. Xing, et al., Transactions of the CSAM, 40, 56 (2009)

13. L. Tao, H.R. Yin, L. Jing, et al., Journal of South China Agricultural University, 37, 126 (2016)

14. S. Maitraye, B. Dana, S.Ravendra and R. Rohit, Processes, 89 (2014)

15. L. Bart, A. Thomas, T. Engelbert, et al., Computers and Electronics in Agriculture, 24 (2014)

16. Z. Xuan and L.V. Quoc, Mech Mater, 115 (2000)

17. I. Keppler, L. Kocsis, I. Oldal, et al., Advanced Powder Technology, 824 (2012)

18. L.Y. Qin, Z.M. Quan, L. Fei, et al., Transactions of the CSAM, 47, 65 (2016)

19. L.Q. Xi, Z.P. Ling, L.Y. Tao, et al., Transactions of the CSAM, 45, 109 (2014)

20. L.H. Chang, L.Y. Ming, T. Zhong, et al., Transactions of the CSAE, 27, 117 (2011)

21. L.P. Van, E. Tijskens, E. Dintwa, et al., Powder Technology, 190, 348 (2009)

22. V. Aleshin and K. Van Den Abeele, International Journal of Non-Linear Mechanics, 15 (2013)

23. Y. Shimizu and P. Cundall, J Eng Mech-Asce, 127, 864 (2001)

24. A.O. Raji and J.F. Favier, Journal of Food Engineering, 359 (2004)

25. L. Yang, H.Y. Long, J.F. Guo, et al., Acta Phys. Sin., 64, 114501 (2015)

26. Y.J. Qun, S.Y. Fang, N.X. Ttang, et al., Transactions of the CSAE, 24, 105 (2008)

27. H.Z. Wu, M.W. Peng, X.J. Long and L.Q. Hui, Journal of south China agricultural university, 37, $109(2016)$

28. Z. Zhan, W.Y. Fang, Y.J. Jun and T. Zhong, Computers and Electronics in Agriculture, 25 (2015)

29. L.Q. Hui, G.X. Jun and Z.Z. Hong, Simulation and Experiment of Seed-filling Performance of Pneumatic Cylinder Seed-metering Device for Panax notoginseng, 47, 27 ( 2016)

30. S.L. Rong, W.J. Min, S. Wei, et al., Transactions of the CSAE, 30, 40 (2014) 